Pediatric ADS is rare, a previous study in Canadian children finding an overall ADS incidence of 0.9 per 100,000.(Banwell B et al. Neurology 2009;72:232-239).

Risk of MS in children is dependent on genetic factors (presence of HLADRB $1 * 15$ genotype) and environmental triggers (previous infection with Epstein-Barr virus, and low serum 25-hydroxyvitamin D concentrations).(Banwell B et al. Lancet Neurol 2011;10(5):436-445). The increased risk conveyed by HLA-DRB1*15 alleles relates to chronic MS rather than ADS in general (Disanto G et al. Neurology 2011;76(9):781-786). Whereas remote infection with Ebstein-Barr virus is associated with an increased risk of pediatric MS, some viruses (cytomegalovirus) may lower MS susceptibility. HSV-1 remote infection does not increase odds of MS but it has a strong interaction with HLA-DRB1 in predicting MS. (Waubant E et al. Neurology 2011;76(23):1989-1995).

\title{
OPTIC NEURITIS, ABNORMAL MRI AND OUTCOME
}

Researchers at Birmingham Children's Hospital, and Great Ormond Street and Evelina Children's Hospitals, London, UK followed 44 children (female/male ratio 1.8; median age 10.9 years) with a first-episode optic neuritis for a median of 1 year (mean, 1 year 10 months). Maximal visual deficit was severe $(<6 / 60)$ in $77 \%$. Recovery was complete in $70 \%$. At follow-up, $32 \%$ were diagnosed with MS (11/44) or neuromyelitis optica (NMO) (3/44). Cumulative probability of developing MS or NMO by 2 years after optic neuritis onset was 0.45 . Gender, age, unilateral/bilateral optic neuritis, visual acuity severity, and CSF oligoclonal bands were non-predictive. Strong predictors of MS or NMO were relapsing optic neuritis $(\mathrm{p}<0.001)$ and an initial abnormal MRI $(>1$ brain T2 hyperintense lesion) $(\mathrm{p}<0.001)$. (Absoud M, Cummins C, Desai N, et al. Childhood optic neuritis clinical features and outcome. Arch Dis Child Sept 2011;96:860-862). (Respond: Dr Michael Absoud, Clinical Research Fellow, Institute of Child Health, Birmingham Children's Hospital, Steelhouse Lane, Birmingham B4 6NH. E-mail: michaelabsoud@childdemyelination.org.uk).

COMMENT. Pediatric acute onset optic neuritis has a good initial prognosis. An abnormal initial MRI or relapsing optic neuritis is predictive of MS or NMO.

\section{LANGUAGE DISORDERS}

\section{PRENATAL FOLIC ACID SUPPLEMENTS AND REDUCED RISK OF SEVERE LANGUAGE DELAY}

The prospective Norwegian Mother and Child Cohort Study of pregnant women between 1999 and 2008 was used by researchers at the Norwegian Institute of Public Health, Oslo to examine the association between mothers' use of prenatal folic acid supplements and risk of severe language delay in the children at age 3 years. Language development assessment was based on follow-up questionnaires returned by $61 \%$ of mothers in 2010. Children with only 1-word or unintelligible utterances were rated as having severe language delay. Children who could only produce 2 - to 3 -word phrases 
were rated as having moderate language delay, an outcome that is less readily interpreted than severe delay. Children producing fairly complete sentences were rated as having no language delay. Among a total of 38,954 children, $204(0.5 \%)$ had severe language delay. Of the reference group $(24 \%)$ who took no dietary supplements, $81(0.9 \%)$ had severe language delay. (In Norway, foods are not fortified with folic acid).

Relative risks of severe language delay by estimating odds ratios for 3 patterns of exposure to folic acid were (1) $0.9 \%$ [OR 1.04] in children of mothers taking other supplements but no folic acid; (2) $0.4 \%$ [0.55] for those taking folic acid only; and (3) $0.4 \%[0.55]$ for those taking folic acid and other supplements. The adjusted ORs and risk of moderate language delay for the three groups were $1.04,0.82$, and 0.79 , respectively. Maternal use of folic acid supplements in early pregnancy, 4 weeks before to 8 weeks post-conception, was associated with a reduced risk of severe language delay in children at age 3 years. Maternal reports and Vineland test scores for receptive and expressive communication skills in a subsample of 425 children were highly correlative. A higher proportion of children with severe language delay had not yet attained expected motor skills at 18 months and 3 years, but use of folic acid supplements was not correlated with attainment of gross motor skills. (Roth C, Magnus P, Schjolberg S, et al. Folic acid supplements in pregnancy and severe language delay in children. JAMA October 12, 2011;306(14):1566-1573). (Respond: Christine Roth MSc ClinPsyD, Division of Mental Health, Norwegian Institute of Public Health, PO Box 4404, Nydalen, 0403 Oslo, Norway. E-mail: Christine.roth@fhi.no).

COMMENT. Periconceptional folic acid supplements are known to reduce risk of neural tube defects (MRC Vitamin Study. Lancet 1991;338(8760):131-137), but the present study is considered the first to demonstrate effects on language development. The average child usually says his first word by 1 year, and delay beyond 18 months may indicate a severe physical, mental or hearing handicap. Failure to put 2 or 3 words together in short phrases by 2 years and sentences by age 3 years is a significant delay in language development. Adequate hearing is the first requisite for normal language development. If the hearing is normal, the differential diagnosis includes congenital dysphasia, mental retardation, and infantile autism. The brain MRI, fMRI (de Guibert C et al. Brain 2011;134:3044-3058) or pathological studies (Cohen M et al. Ann Neurol 1989;25:567-570) may uncover subtle cerebral anomalies. An EEG may be indicated to rule out an acquired epileptic aphasia (Landau-Kleffner syndrome) that develops at 2 to 5 years of age.(Cockerell I et al. Epilepsy Behav 2011;21:153-159) The cause of severe language delay associated with periconceptional folic acid deficiency is unclear. Prenatal effects on brain development (Milunsky A et al. JAMA 1989;262:2847-2852), and epigenetic dysregulation of gene expression are proposed possible mechanisms.. (Jaenisch R et al. Nat Genet 2003;33(suppl):245-254).

\section{ABNORMAL FUNCTIONAL LATERALIZATION OF LANGUAGE IN DEVELOPMENTAL DYSPHASIA}

Researchers at University Hospital, Rennes, France compared functional MRI studies of 21 children with developmental dysphasia and matched controls using a panel of four language tests including auditory and visual tasks. Children with specific 